\title{
Neue Herausforderungen für eine integrierende Stadtentwicklung
}

https://doi.org/10.2478/rara-2019-0044

Der Ansatz der integrierenden Stadtentwicklung knüpft an die vor allem in den 1990er-Jahren geführte Diskussion um Konzepte der "local governance" an, bezieht sich jedoch auch auf Ansätze der „democratic network governance", wenn auf der Basis von lokalen Netzwerken Maßnahmen geplant und umgesetzt werden, die in Richtung einer solidarischen und integrierenden Stadtgesellschaft wirken (sollen). Integrierende Stadtentwicklung geht im Gegensatz zur „integrierten Stadtentwicklung“, wie sie zum Beispiel im Rahmen der Programme zur "Sozialen Stadt" in Deutschland umgesetzt wurde und wird, nicht nur von einem Aufbrechen der Versäulung innerhalb der administrativen Strukturen und dem Einsatz von intermediären Akteuren zur Vermittlung zwischen politisch-administrativem System und Akteuren vor Ort aus. Sie bezieht bereits bei der Formulierung von Zielvorstellungen und hiermit verbundenen Fragestellungen Akteure des politisch-administrativen Systems, der Zivilgesellschaft, Sozialwirtschaft und Wirtschaft mit ein. Die Rolle der Wissenschaft besteht in diesem Zusammenhang darin, die erarbeiteten Fragestellungen aufzugreifen, die jeweiligen Ausgangslagen bzw. lokalen Kontexte zu analysieren, unterschiedliche Perspektiven auf jeweils wahrgenommene Herausforderungen und Potenziale der Entwicklung aufzudecken sowie den Wissenstransfer und die Auseinandersetzung zwischen Wissenschaft und Praxis wie auch zwischen den stadtgestaltenden Akteuren untereinander zu unterstützen.

Das vorliegende Schwerpunktheft der Zeitschrift „Raumforschung und Raumordnung | Spatial Research and Planning" stellt Forschungen zur integrierenden Stadtentwicklung vor, die unterschiedliche lokale Kontexte untersuchen. Obwohl ausschließlich Entwicklungen in deutschen Städten behandelt werden, lassen sich einzelne Aspekte, nicht zuletzt aufgrund sich ähnelnder Herausforderungen, auf andere europäische Kontexte übertragen. Die Ergebnisse der Forschungen zeigen, dass als eine der größten Herausforderungen einer integrierenden, das heißt sozial schwache Gruppen in ihrer gesellschaftlichen Teilhabe unterstützenden, Stadtentwicklung und Stadtplanung nach wie vor die wachsende soziale Segregation und Konzentration von Problemlagen in bestimmten Quartieren gesehen wird. Diese seit Längerem bestehende Herausforderung beispielsweise in Bezug auf die Steuerung des Wohnungsmarktes stellt sich jedoch neu, wenn gesellschaftliche Ereignisse wie die vermehrte Ankunft von Geflüchteten seit dem Jahr 2015 die Kommunen zu veränderten Reaktionen zwingt. Im Zuge der vorgestellten Forschungen geraten Ansatzpunkte kommunaler Integrationspolitiken ins Blickfeld, die bisher jenseits des Aufgabenfeldes der Integration diskutiert werden. Sichtbar werden Integrationspotenziale, die nur im Zusammenspiel von räumlicher und sozialer Dimension wirksam werden können.

Vier der vorliegenden Beiträge befassen sich mit neuen Herausforderungen, die durch den Zuzug von Geflüchteten in unterschiedlichen kommunalen Handlungsfeldern entstanden. Im Zentrum steht dabei die Frage des Wohnens und des sozialen Zusammenlebens in den Quartieren bzw. Kommunen. Welche Veränderungen innerhalb von politisch-administrativen Strukturen, aber auch in Bezug auf die individuellen Einstellungen der Bewohnerinnen und Bewohner sind im Zuge der festgestellten Stadtentwicklungen zu konstatieren? Die anschließenden Beiträge stellen Wissen generierende Forschungen sowie Vorgehensweisen zur Entwicklung einer integrierenden Stadtentwicklung vor. Ihre Ergebnisse hinterfragen das auf der Grundlage

${ }^{*}$ Corresponding author: Prof. Dr. Heike Herrmann, Hochschule Fulda, Fachbereich Sozialwesen, Leipziger Straße 123, 36037 Fulda, Deutschland, E-mail: heike.herrmann@sw.hs-fulda.de 
einer steigenden Segregation entstandene Leitbild der sozialen Mischung, wenn das Zusammenspiel von (den individuellen Lebenslagen Benachteiligter entsprechenden) Bedürfnissen respektive Ressourcen und den Möglichkeiten („Opportunities“) des Quartiers im Fokus der Untersuchungen steht. Quartiersentwicklung kann damit nicht einem allgemeinen Leitbild folgen. Es braucht kontextspezifische Strukturen und Verfahren der Vernetzung der jeweils zu beteiligenden Akteure.

Henning Nuissl, Valentin Domann und Susen Engel sehen anhand dreier empirischer Fallstudien in unterschiedlichen Räumen (Ankunftsquartier, Großwohnsiedlung und Kleinstadt) ein sich neu formierendes Feld lokaler Integrationspolitik entstehen, das sich als Teil einer integrierenden Stadtentwicklung verstehen lässt. Auslöser sind dabei die im Verlauf der fluchtbedingten Zuwanderung entstehenden Herausforderungen der Unterbringung und Integration von Geflüchteten. In Anlehnung an das Modell von Ager und Strang (2008) konzeptualisieren die Autorin und die Autoren Integration als einen reziproken Prozess. Dieser ist eng verbunden mit den auch außerhalb der klassischen Integrationspolitik, das heißt in anderen lokalen Handlungsfeldern, wirksam werdenden Maximen der wechselseitigen Anerkennung, der Absicherung (in Zeiten der „politics of uncertainty") und der allgemeinen kommunalen Zielvorstellung der sozialen Kohäsion. Sie zeigen in ihrem Beitrag sehr deutlich, wie unterschiedliche sozialräumliche Bedingungen, aber auch lokale diskursive Rahmungen der genannten Herausforderungen jeweils unterschiedliche Integrationsmodi befördern bzw. andere behindern können.

Handlungslogiken kommunaler Akteure der Integrationspolitik stehen auch im Mittelpunkt des Beitrags von Claus Wiegandt, Stefanie Föbker, Daniela Imani, Carmella Pfaffenbach, Francesca Adam und Günther Weiss. Ihre vergleichende Untersuchung eines großstädtischen sozialräumlichen Kontextes mit den klein- und mittelstädtisch geprägten Gemeinden eines Landkreises zeigt, dass die Kommunen durch das Ausschöpfen von Spielräumen einen nicht unwesentlichen Einfluss auf die Integration von Geflüchteten nehmen können, auch wenn auf der Ebene des Bundes und der jeweiligen Bundesländer über die Gesetzgebung ein Handlungsrahmen gesetzt wird. Auf der Kommunalebene wird die Vernetzung der an den Integrationspolitiken beteiligten Akteure als Schlüssel für eine erfolgreiche Integrationsarbeit gesehen. Die Autorinnen und Autoren weisen zudem auf Spannungen und Konflikte hin, die durch unterschiedliche Zeithorizonte, Arbeitsabläufe, aber auch Handlungsziele der befragten Akteure entstehen.
Die an der integrierenden Stadtentwicklung beteiligten Netzwerke unterliegen unterschiedlichen Handlungslogiken: Während kommunales Verwaltungshandeln sich in einem klaren, von rechtlichen Vorgaben bestimmten Rahmen bewegt, reagieren Akteure der Zivilgesellschaft spontan und flexibel auf neue Anforderungen. Auch Zielformulierungen bedürfen aufgrund eines unterschiedlichen Verständnisses von Integration und angestrebten Integrationsverläufen der Auseinandersetzung.

Jürgen Friedrichs, Felix Leßke und Vera Schwarzenberg nahmen die in dem Zeitraum 2015 bis 2018 praktizierte Unterbringung von Geflüchteten zum Anlass, nach der Einstellung und den Vorurteilen von Anwohnerinnen und Anwohnern gegenüber den Geflüchteten und den Flüchtlingsunterkünften als solche zu fragen. In ihrem Beitrag stellen sie Ergebnisse einer repräsentativen Befragung von Anwohnerinnen und Anwohnern sowie Geflüchteten in jeweils zwei Wohngebieten dreier Städte vor, in denen Flüchtlingsunterkünfte eingerichtet wurden. Die Ergebnisse zeigen insgesamt eine - trotz anfänglicher Proteste - positive Einstellung gegenüber den Geflüchteten und den Unterkünften, wobei diese in Gebieten mit höherem Status deutlich positiver ausfällt. Über Fragen nach der Auseinandersetzung, Akzeptanz und Solidarität mit den Geflüchteten erhoben die Autorin und die Autoren auch Faktoren des sozialen Zusammenhalts. Vor dem Hintergrund, dass die Befragten der Untersuchung mit dem Zuzug der Geflüchteten zahlreiche Ängste verbinden, und der Tatsache, dass die Akzeptanz einer Flüchtlingsunterkunft stark vom (politischen) Beteiligungsprozess der Bewohnerinnen und Bewohner und der Art der Unterbringung (zentral oder dezentral in Wohnungen) abhängig ist, sind die Ergebnisse auch ein Plädoyer für eine integrierende Stadtentwicklung, die den Diskurs mit den Bewohnerinnen und Bewohnern sucht.

Auch Sebastian Kurtenbach befasst sich mit neuen Herausforderungen einer Stadtentwicklungspolitik, die sich durch den Zuzug von Geflüchteten entwickelt haben. Er konstatiert für seinen Untersuchungskontext, Bautzen in Sachsen, den Verlust kollektiver und individueller Sicherheiten. Mit Bezug auf Ansätze zu den Kontexteffekten bestimmter sozialräumlicher Konstellationen (welche er auf die Gesamtstadt überträgt), Forschungen zum Rechtspopulismus (die eine Legitimation von Anfeindungen gegenüber einer bestimmten Gruppe und eine Normverschiebung feststellen) und raumbezogenen Studien zur Menschenfeindlichkeit sieht der Autor einen spezifischen Kontext der Ablehnung und Ausgrenzung entstehen. Dieser ist verbunden mit einer von den Befragten wahrgenommenen Erosion des stadtgesell- 
schaftlichen Zusammenhalts, einer Verschiebung von Deutungshoheiten sowie unterschiedlichen Arenen der Exklusion. Der Beitrag wirft viele Fragen auf. So unter anderen die, ob und wie die beschriebenen Arenen der Exklusion hätten verhindert werden können.

In Adaption des kanadischen „Middle Years Development Instrument“ (MDI) und gestützt auf (internationale) Untersuchungen zum Wohlbefinden von Kindern und Jugendlichen verfolgen Sören Petermann, Peter Strohmeier und Katharina Yaltzis gemeinsam mit lokalen Akteuren aus der Bildungs- und Sozialpolitik das Ziel, ein kleinräumiges Monitoring-System zum Wohlbefinden von Kindern und Jugendlichen zu entwickeln. Ihre Forschung konzentriert sich auf eine besondere Form von Kontexteffekten, wenn sie in Anlehnung an das Modell zur kindlichen Entwicklung von Bronfenbrenner (1981) annehmen, dass die Familie, die Schule und die Wohnumgebung als ,Kontexte' einen wesentlichen Einfluss auf das Wohlbefinden von Kindern und Jugendlichen nehmen. Ziel der kooperativen Entwicklung eines Monitoring-Instruments des "local governance" und der hiermit angestoßenen Prozesse war es, Ansatzpunkte dafür zu erhalten, wie die erhobenen Kontexte zu gestalten sind, sodass das Wohlbefinden der Kinder und Jugendlichen gesteigert werden kann.

Nina Schuster und Anne Volkmann befassen sich in ihrer qualitativen Forschung mit dem Einfluss des Quartiers auf die Möglichkeiten und Wahlfreiheiten der Lebensgestaltung von Bewohnerinnen und Bewohner eines im klassischen Sinne als benachteiligt charakterisierten Quartiers. Sie richten ihren Blick auf das Zusammenspiel von individuellen Ressourcen und Bedürfnissen mit den - unter anderem im Quartierskontext - vorhandenen Möglichkeiten der Bedürfnisbefriedigung. Mit dem Fokus auf die nach Alter, Geschlecht und unterschiedlichem Kapital im Sinne Bourdieus geprägten Lebenslagen der Bewohnerinnen und Bewohner ist es nicht das Quartier an sich, sondern das Quartier als ein Element der Möglichkeiten der Bedürfnisbefriedigung in einer spezifischen Lebenslage (die Autorinnen schreiben: die Lebenslage selbst), die als benachteiligend zu bezeichnen ist. Ein wesentlicher Faktor im Zusammenspiel individueller Ressourcen und der Möglichkeiten der Bedürfnisbefriedigung ist die Mobilität. Mobilitätseinschränkungen stellen bei fehlenden Möglichkeiten der Bedürfnisbefriedigung innerhalb des Quartiers eine Benachteiligung dar. Sie stärken mit diesem Ergebnis den internationalen Ruf nach neuen, im Netzwerk der lokalen Akteure zu entwickelnden Mobilitätskonzepten.

Andreas Farwick, Heike Hanhörster, Isabel Ramos Lobato und Wiebke Striemer fokussieren in ihrer For- schung ebenfalls die den Bewohnerinnen und Bewohnern zur Verfügung stehenden Ressourcen. Welche Bedeutung haben die Ebene des Quartiers und die kleinräumige Nachbarschaft für den Ressourcentransfer? Hintergrund der Forschung ist die Herausforderung einer sich verschärfenden sozialen Segregation und ihrer Folgen. In Anlehnung an die Kapitaltheorie von Bourdieu und auf der Grundlage von Netzwerkforschungen in Bezug auf soziale Ressourcen untersuchen die Autorinnen und der Autor in einem Methodenmix zwei Dortmunder Quartiere und hier bestimmte Orte der sozialen Interaktion und des Ressourcentransfers (,foci'). Sie unterscheiden dabei den Support durch Kontakte und Netzwerke im unmittelbaren Wohnumfeld und darüber hinaus. Ihre Ergebnisse zeigen, dass eine soziale Mischung im Quartier nicht automatisch die Möglichkeit des sozialen Aufstiegs über statusübergreifende Kontakte bedeutet. Auch homogene Netzwerke einkommensarmer Haushalte können "Getting-ahead“Ressourcen zur Verfügung stellen. Jedoch wirkt sich soziale Segregation im Hinblick auf die Integration in den Arbeitsmarkt negativ aus, sodass diesbezüglich institutionelle Ressourcen zur Verfügung zu stellen sind.

Die beiden letztgenannten Beiträge sind ein Plädoyer dafür, Quartiersentwicklung im Sinne einer integrierenden Stadtentwicklung sowohl im Quartier selbst (Erweiterung der "Opportunities") als auch darüber hinaus (Förderung der Mobilität) zu betreiben und dabei die Bedürfnisse bzw. Lebenslagen der Bewohnerinnen und Bewohner bzw. diese selbst mit einzubeziehen. Eine „local democratic network governance“ im Sinne einer integrierenden Stadtentwicklung muss sich mit verschiedenen sozialräumlichen Kontexten auf unterschiedlichen Ebenen („skales“) auseinandersetzen. Die in diesem Heft vorgestellten Forschungen machen deutlich, dass sich neu stellende Herausforderungen Impulse für veränderte Strukturen und Verfahren der integrierenden Stadtentwicklung hervorbringen können. Die Zukunft wird zeigen, ob diese in eine neue Normalität transformiert werden können.

\section{Literatur}

Ager, A.; Strang, A. (2008): Understanding Integration. A Conceptual Framework. In: Journal of Refugee Studies 21, 2, 166-191. doi: 10.1093/jrs/fen016

Bronfenbrenner, U. (1981): Die Ökologie der menschlichen Entwicklung: Natürliche und geplante Experimente. Stuttgart. 